\title{
Nonrandom Association of Avirulence Loci in Four Disease Resistance Gene System II. Replacement between Host Groups Having Different Number of Susceptibility Genes
}

\author{
Shigehisa KIYosaWA*,***, Donna PuRBA**,+, Md. Shamsher Ali**,+ \\ Yasushi OKINAKA*,tt, Tsutomu SHIMIZU** and Akihiko SAITO**
}

\begin{abstract}
The influence of replacement between host genotypes with different S groups (groups with a different number of resistance or susceptibility genes) on the types and patterns of nonrandom associations between avirulence loci in the pathogen was examined by a method of simulation. Through all combinations of $\mathrm{S}$ groups, nonrandom association patterns are largely influenced by various factors, and degrees of influence are in the following order. 1) Direction of transfer of values from donor to receptor (replacement of varieties with different genotypes from old to new), 2) combinations of S groups (groups having the different number of susceptibility genes), 3) fitness values given to virulence genes when various values with the same average are given, 4) generations of vegetative reproduction, and 5) the number of resistance loci with complementary resistance and susceptibility genes. When a large area was replaced by a new host genotype with one or three different genes, half of the possible six nonrandom associations were cross (C)-type and the remainder were noncross (N)-type. In these cases, combinations of an avirulence gene corresponding to a resistance gene in the new host genotype with other avirulence loci in the pathogen show $\mathrm{N}$-type nonrandom associations. If a receptor has two resistance genes, combinations including one of avirulence loci corresponding to resistance genes in the receptor induce C-type nonrandom associations and combinations including the two loci show $\mathrm{N}$ type. Accordingly, the ratio of $\mathrm{N}: \mathrm{C}$ was $2: 4$. When a receptor has three resistance genes, the ratio is $3: 3$. This can explain why two types were found in fields where two or more statistically significant nonrandom associations were found.
\end{abstract}

(Received May 9, 1994 ; Accepted October 4, 1995)

Key words : population genetics, nonrandom association, virulence analysis, host, pathogen, fitness.

\section{INTRODUCTION}

In the previous paper6), we discussed the determination of nonrandom association types or patterns in cases where a host genotype was changed to another genotype having the same number of susceptibility genes (for example, from resistance genotype $\mathrm{AABB}++\mathrm{DD}$ to $\mathrm{AA}++\mathrm{CCDD}$, hereafter referred to as $\mathrm{AB}+\mathrm{D}$ and $\mathrm{A}+$ $\mathrm{CD}$ ). In the paper, it was demonstrated that the nonrandom association types (interaction between two loci in four avirulence loci, a, b, c and d) or patterns [a set of six interactions, $\mathrm{a}-\mathrm{b}, \mathrm{a}-\mathrm{c}, \mathrm{a}-\mathrm{d}, \mathrm{b}-\mathrm{c}, \mathrm{b}-\mathrm{d}$ and $\mathrm{c}-\mathrm{d}$ )] are determined by the donor in some cases and by the receptor in other cases. When a donor determines the nonrandom association type, pairs of two resistance genes and two susceptibility genes induce cross-type (C) in nonrandom associations. Other pairs, a resistance and a susceptibility gene, induce noncross-type $(\mathrm{N})$ nonrandom associations. In the cases where the type or pattern is determined by a receptor, its type is the noncross-type in the former pairs and cross-type in the latter. Where

\footnotetext{
* Tsukuba Research Laboratories, Kyowa Hakko Co., Tsukuba 305, Japan＼cjkstart協和発酵工業株式会社筑波研究所

** Tsukuba International Agricultural Training Center, Tsukuba 305, Japan＼cjkstart筑波国際農業研修センター

*** Present address : 31-8, Sakuragaoka, Kukizaki-machi, Inashiki-gun, Ibaraki 300-12, Japan 現在 : 茨城県稲敷郡茎崎 町桜が丘 $31-8$

† Present address: Directorate of Food Crops Production Development, J1. AUP 3, Pasar Minggu, Jakarta Selatan, Indonesia

†† Present address : Bangladesh Rice Research Institute, Gazier 1701, Bangladesh

t+t Present address : Hokkaido University, Sapporo 060, Japan＼cjkstart現在：北海道大学
} 
all six pairs between avirulence loci could be explained by the types expected from the donor or receptor these were called donor $(\mathrm{D})$ or receptor $(\mathrm{R})$ pattern, respectively. When the different pairs in the same number (for example 5) can be explained by the donor or receptor, respectively, it was called $5 \mathrm{DR}$ pattern. When pairs explained by the donor (for example 4) were more than those by the receptor, it was called $4 \mathrm{D}$ pattern and the reverse ones called $4 \mathrm{R}$ pattern.

In the present paper, we will report cases in which a host genotype is replaced by another genotype with a different number of susceptibility genes.

\section{MATERIALS AND METHODS}

The same methods as the previous paper ${ }^{6}$ were followed except the use of simulation data on interaction between groups (S) with different numbers of susceptibility genes.

\section{RESULTS AND DISCUSSION}

\section{Replacement between host groups having differ- ent numbers of susceptibility genes}

At first, $\mathrm{S}_{1} \rightarrow \mathrm{S}_{2}$ transfers (replacement of varieties with one susceptibility gene with varieties having two susceptibility genes) were analyzed (Table 1).

Generally, there are predominant $\mathrm{R}$ patterns (patterns with a larger number of nonrandom association types expected from the receptor than the donor) under the standard conditions where 0.75 was given to all four virulence genes. Where only one locus is complementary for a resistance gene and a susceptibility gene between donor and receptor (for example, $\mathrm{A}+\mathrm{CD} / \mathrm{A}++\mathrm{D}$ ), $6 \mathrm{R}$ type of non-random associations occur, and where three genes are complementary $(\mathrm{A}+\mathrm{CD} / \mathrm{AB}++)$, $5 \mathrm{R}$ type occurs.

In the reverse direction of replacement of host genotypes (Table 2), D patterns are predominant in early generations of vegetative reproductions after the replacement of host genotypes. Where three loci for resistance are complementary, nonrandom association patterns change to receptor types and where only one locus is complementary, there is no change in the pattern at all.

Under nonstandard conditions, donor patterns appear in early generations even in $\mathrm{S}_{1} \rightarrow \mathrm{S}_{2}$ (Table 1 ) where receptor patterns are present exclusively. These patterns change to receptor patterns in later generations in combinations with three complementary loci for resistance. The remaining combinations with one complementary locus for resistance do not show a change in patterns.

Under nonstandard conditions in $\mathrm{S}_{2} \rightarrow \mathrm{S}_{1}$, change in the patterns occurs (Table 2); this indicates that the nonrandom association patterns are influenced by the fitness values given to the virulence genes. All of D (donor) patterns which are found at the 10th generation change to $\mathrm{R}$ (receptor) patterns at the 200th generation.

It has been noted that there are $5 \mathrm{R}$ or $5 \mathrm{D}$ patterns in half combinations in both $\mathrm{S}_{1} \rightarrow \mathrm{S}_{2}$ and $\mathrm{S}_{2} \rightarrow \mathrm{S}_{1}$. These patterns appear where three loci are complementary

Table 1. Influence of interaction between two avirulence loci on type of nonrandom associations $\left(\mathrm{S}_{1} \rightarrow \mathrm{S}_{2}\right)^{\mathrm{d})}$

\begin{tabular}{|c|c|c|c|c|c|c|}
\hline \multirow{2}{*}{$\begin{array}{c}\text { Donor } \\
\text { (Genotype) }\end{array}$} & \multicolumn{6}{|c|}{ Receptor } \\
\hline & $\begin{array}{c}Q_{6}(\mathrm{AB}++) \\
{[\mathrm{NCCCCN}]}\end{array}$ & $\begin{array}{c}Q_{7}(\mathrm{~A}+\mathrm{C}+) \\
{[\mathrm{CNCCNC}]}\end{array}$ & $\begin{array}{c}Q_{8}(+\mathrm{BC}+) \\
{[\mathrm{CCNNCC}]}\end{array}$ & $\begin{array}{c}Q_{9}(\mathrm{~A}++\mathrm{D}) \\
{[\mathrm{CCNNCC}]}\end{array}$ & $\begin{array}{l}Q_{10}(+\mathrm{B}+\mathrm{D}) \\
{[\mathrm{CNCCNC}]}\end{array}$ & $\begin{array}{l}Q_{11}(++\mathrm{CD}) \\
{[\mathrm{NCCCCN}]}\end{array}$ \\
\hline $\begin{array}{l}Q_{2}(\mathrm{ABC}+) \quad \mathrm{E}^{\mathrm{a})} \\
{[\mathrm{C} \mathrm{CNCNN}]^{\mathrm{b})}}\end{array}$ & 1. $\underline{\mathrm{NCCC} \mathrm{C} \mathrm{N}}^{\mathrm{c})}$ & 2. $\mathrm{CNCCNC}$ & 3. $\underline{\mathrm{CCNNCC}}$ & 4. $\underline{\mathrm{CCNC}} \underline{\mathrm{CC}}$ & 5. $\underline{\mathrm{CCCNC}}$ & 6. $\mathrm{CCCCCN}$ \\
\hline $\begin{array}{l}Q_{3}(\mathrm{AB}+\mathrm{D}) \quad \mathrm{E} \\
{[\mathrm{C} \mathrm{NCNCN]}}\end{array}$ & 7. $\mathrm{NCCCCN}$ & 8. $\underline{\mathrm{CNCCCC}}$ & 9. $\underline{\mathrm{CCC}} \underline{\mathrm{NCC}}$ & 10. $\mathrm{CCNNCC}$ & 11. $\mathrm{CNCCNC}$ & 12. $\mathrm{CCCCN}$ \\
\hline $\begin{array}{l}Q_{4}(\mathrm{~A}+\mathrm{CD}) \quad \mathrm{E} \\
{[\mathrm{NCCNNC}] \mathrm{U}}\end{array}$ & 13. $\frac{\mathrm{NCCCCC}}{\mathrm{NCCNNC}}$ & 14. $\mathrm{CNCCNC}$ & $\begin{array}{l}\text { 15. } \frac{\mathrm{CCCNCC}}{\mathrm{CCC} N \mathrm{NNC}} \\
\underline{\mathrm{CCCNCC}}\end{array}$ & 16. $\mathrm{CCNNCC}$ & 17. $\underline{\mathrm{CCCNC}}$ & 18. $\mathrm{NCCCCN}$ \\
\hline $\begin{array}{l}Q_{5}(+\mathrm{BCD}) \quad \mathrm{E} \\
{[\mathrm{NNNCCC}] \mathrm{U}}\end{array}$ & $\begin{array}{l}\text { 19. } \frac{\mathrm{NCCCCC}}{\mathrm{NCNCCC}} \\
\underset{N C C C C C}{\mathrm{NCCC}}\end{array}$ & $\begin{array}{l}\text { 20. } \frac{\mathrm{CNCCCC}}{\mathrm{CNNCCC}} \\
\underline{\text { N NCCC }}\end{array}$ & 21. $\mathrm{CCNNCC}$ & 22. $\underline{\mathrm{CCNC}} \underline{\mathrm{CC}}$ & 23. $\mathrm{CNCCNC}$ & 24. $\mathrm{NCCCCN}$ \\
\hline
\end{tabular}

a) $\mathrm{E}:$ Standard conditions: the case where equal fitness value 0.75 was given to all virulence genes. The patterns obtained can be explained as receptor type, too. Cases where the pattern is completely same as patterns under nonstandard conditions.

$\mathrm{U}$ : Nonstandard conditions. Unequal fitness values, $0.9,0.8,0.6$ and 0.7 were given to $+^{\mathrm{d}},+^{\mathrm{c}},+^{\mathrm{b}}$ and $+^{\mathrm{a}}$, respectively.

b) [ ] indicates patterns from the donor and receptor, respectively.

c) and andicate that patterns obtained by simulations can be explained by influence from the receptor and donor, respectively.

d) In some fields, an old genotype $\left(\mathrm{S}_{1}\right)$ was replaced by a new genotype $\left(\mathrm{S}_{2}\right) . Q_{2}, Q_{3}, Q_{4}$ and $Q_{5}$ are components of $\mathrm{S}_{1}$, and $Q_{6}, Q_{7}, Q_{8}, Q_{9}, Q_{10}$ and $Q_{11}$ are components of $S_{2}$. For example, $Q_{2}$ and $Q_{6}$ are frequencies of host genotypes, AABBCC ++ and $\mathrm{AABB}++++$, respectively (See Kiyosawa et al. $\left.{ }^{6}\right)$. 
Table 2. Influence of interaction between two avirulence loci on type of nonrandom associations $\left(\mathrm{S}_{2} \rightarrow \mathrm{S}_{1}\right)$

\begin{tabular}{|c|c|c|c|c|c|}
\hline \multirow{2}{*}{$\begin{array}{c}\text { Donor } \\
\text { (Genotype) }\end{array}$} & & \multicolumn{4}{|c|}{ Receptor } \\
\hline & & $\begin{array}{c}Q_{2}(\mathrm{ABC}+) \\
{[\mathrm{NNC} \mathrm{NCC}]}\end{array}$ & $\begin{array}{c}Q_{3}(\mathrm{AB}+\mathrm{D}) \\
{[\mathrm{NC} N \mathrm{NC}]}\end{array}$ & $\begin{array}{c}Q_{4}(\mathrm{~A}+\mathrm{CD}) \\
{[\mathrm{C} N \mathrm{NC} \text { C }]}\end{array}$ & $\begin{array}{c}Q_{5}(+\mathrm{BCD}) \\
{[\mathrm{CCCNNN}]}\end{array}$ \\
\hline \multirow{3}{*}{$\begin{array}{l}Q_{6}(\mathrm{AB}++) \\
{[\mathrm{C} N N N N C]}\end{array}$} & $\mathrm{E}$ & C NNNNC & C NNNNC & CNNNNN & CNNNNN \\
\hline & & & & CNNCCN & $\mathrm{CCCNNN}$ \\
\hline & $\mathrm{U}$ & & & $\overline{\mathrm{CNNCCN}}$ & $\overline{\mathrm{CCCNNN}}$ \\
\hline \multirow{4}{*}{$\begin{array}{l}Q_{7}(\mathrm{~A}+\mathrm{C}+) \\
{[\mathrm{NC} N \mathrm{NCN}]}\end{array}$} & $\mathrm{E}$ & NCNNCN & NCNNNN & NCNNCN & NCNNNN \\
\hline & & & NCNCNC & & CCCNNN \\
\hline & $\mathrm{U}$ & & & & $\overline{\mathrm{NCCNNN}}$ \\
\hline & & & & & $\underline{\mathrm{CCCNNN}}$ \\
\hline \multirow{3}{*}{$\begin{array}{l}Q_{8}(+\mathrm{BC}+) \\
{[\mathrm{NNCCNN}]}\end{array}$} & $\mathrm{E}$ & NNCCNN & NNNCNN & NNNC NN & NNCCNN \\
\hline & & & NCNCNC & CNNCCN & \\
\hline & $\mathrm{U}$ & & NNNC NC & $\mathrm{CNNCCN}$ & \\
\hline \multirow{4}{*}{$\begin{array}{l}Q_{9}(\mathrm{~A}++\mathrm{D}) \\
{[\mathrm{NNCCNN}]}\end{array}$} & $\mathrm{E}$ & NNC NNN & NNCCNN & NNCCNN & NNC NNN \\
\hline & & NNCNCC & & & $\mathrm{CCCNNN}$ \\
\hline & $\mathrm{U}$ & NNCNNN & & & NNCNNN \\
\hline & & NNC NCC & & & CCCNNN \\
\hline \multirow{4}{*}{$\begin{array}{l}Q_{10}(+\mathrm{B}+\mathrm{D}) \\
{[\mathrm{NCNNCN}]}\end{array}$} & $\mathrm{E}$ & NNNNCN & NCNNCN & NNNNCN & NCNNCN \\
\hline & & NNC NCC & & CNNCCN & \\
\hline & $\mathrm{U}$ & NNNNCN & & NNNCCN & \\
\hline & & NNC NCC & & & \\
\hline \multirow{4}{*}{$\begin{array}{l}Q_{11}(++\mathrm{CD}) \\
{[\mathrm{C} N \mathrm{NNNC}]}\end{array}$} & $\mathrm{E}$ & NNNNNC & NNNNNC & CNNNNC & CNNNNC \\
\hline & & NNC NCC & NCNCNC & & \\
\hline & $\mathrm{U}$ & NNNNNC & NNNNNC & & \\
\hline & & NNC NCC & NCNC NC & & \\
\hline
\end{tabular}

See Table 1 for explanation.

Table 3. Influence of fitness values given in each virulence gene on type of nonrandom associations $\left(\mathrm{S}_{1} \rightarrow \mathrm{S}_{3}\right)$

\begin{tabular}{|c|c|c|c|c|c|}
\hline \multirow{2}{*}{$\begin{array}{c}\text { Donor } \\
\text { (Genotype) }\end{array}$} & & \multicolumn{4}{|c|}{ Receptor } \\
\hline & & $\begin{array}{l}Q_{12}(\mathrm{~A}+++) \\
{[\mathrm{CCCNNN}]}\end{array}$ & $\begin{array}{l}Q_{13}(+\mathrm{B}++) \\
{[\mathrm{CNNCCN}]}\end{array}$ & $\begin{array}{l}Q_{14}(++\mathrm{C}+) \\
{[\mathrm{NC} N \mathrm{NC} \mathrm{NC}]}\end{array}$ & $\begin{array}{l}Q_{15}(+++\mathrm{D}) \\
{[\mathrm{NNCNCC}]}\end{array}$ \\
\hline$Q_{2}(\mathrm{ABC}+)[\mathrm{CCNCNN}]$ & $\mathrm{E}$ & $\overline{\mathrm{CCC}} \overline{\mathrm{NN}}$ & $\overline{\mathrm{C} C} \overline{\mathrm{NCCN}}$ & C $\overline{\mathrm{CNCNC}}$ &  \\
\hline$Q_{3}(\mathrm{AB}+\mathrm{D})[\mathrm{C} \mathrm{NC} \mathrm{NC} \mathrm{N}]$ & $\begin{array}{l}\mathrm{E} \\
\mathrm{U}\end{array}$ & $\overline{\mathrm{CCCNC}}$ & $\overline{\mathrm{CN}} \overline{\mathrm{CCN}}$ & 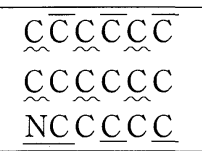 & C $\overline{\text { NCNCC }}$ \\
\hline$Q_{4}(\mathrm{~A}+\mathrm{CD})[\mathrm{NCCNNC}]$ & $\begin{array}{l}\mathrm{E} \\
\mathrm{U}\end{array}$ & $\overline{\mathrm{CCCNNC}}$ & 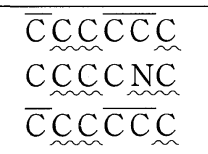 & $\overline{\overline{\mathrm{NC}}} \mathrm{C} \overline{\mathrm{CNC}}$ & $\overline{\bar{N} C \overline{C N C C}}$ \\
\hline$Q_{5}(+\mathrm{BCD})[\mathrm{NNNCCC}]$ & $\mathrm{E}$ & $\mathrm{CCCCCC}$ & C NNCCC & $\mathrm{NCNCCC}$ & NNCCCCC \\
\hline
\end{tabular}

See Table 1 for explanation.

between donor and receptor.

In $\mathrm{S}_{1} \rightarrow \mathrm{S}_{3}$ (Table 3), host-genotype combinations on a diagonal line between $Q_{5}$ and $Q_{15}$ show 3DR patterns. These occur where four resistance loci are complementary. The other host-genotype combinations in which two resistance loci are complementary show 5DR patterns. In these combinations, the influence of the nonstandard conditions does not appear.

In reverse transfer $\left(\mathrm{S}_{3} \rightarrow \mathrm{S}_{1}\right)$ (Table 4$)$, patterns are not different from $S_{1} \rightarrow S_{3}$, although their types in each pair of avirulence loci are the reverse of that of $S_{1} \rightarrow S_{3}$. In these cases, patterns are divided into two groups: One is the $5 \mathrm{DR}$ type and the other is the 3DR type under the standard conditions. The latter occurs where the donor and receptor have four complementary loci. These are seen on the diagonal line from upper-right to lower-left in the table. The others are complementary for two resistance loci. Under the nonstandard conditions, a change in pattern is found from that under the standard conditions (Table 3). Especially, a type (N) marked with $\approx \approx$ is not explained by types $(\mathrm{C})$ expected from donor and receptor. In this case, $\mathrm{C}$ plus $\mathrm{C}$ makes $\mathrm{N}$.

In Table 5 , the cases of $S_{2} \rightarrow S_{3}$ are shown; receptor patterns predominate at the 200th generation. In each 
Table 4. Influence of fitness values given in each virulence gene on type of nonrandom associations $\left(\mathrm{S}_{3} \rightarrow \mathrm{S}_{1}\right)$

\begin{tabular}{|c|c|c|c|c|c|}
\hline \multirow{2}{*}{$\begin{array}{c}\text { Donor } \\
\text { (Genotype) }\end{array}$} & & \multicolumn{4}{|c|}{ Receptor } \\
\hline & & $\begin{array}{c}Q_{2}(\mathrm{ABC}+) \\
{[\mathrm{NNCNCC}]}\end{array}$ & $\begin{array}{c}Q_{3}(\mathrm{AB}+\mathrm{D}) \\
{[\mathrm{NC} N C \mathrm{NC}]}\end{array}$ & $\begin{array}{c}Q_{4}(\mathrm{~A}+\mathrm{CD}) \\
{[\mathrm{C} N \mathrm{~N} C \mathrm{C}]}\end{array}$ & $\begin{array}{c}Q_{5}(+\mathrm{BCD}) \\
{[\mathrm{CCCNNN}]}\end{array}$ \\
\hline$Q_{12}(\mathrm{~A}+++)$ & $\mathrm{E}$ & $\overline{\mathrm{NN}} \overline{\mathrm{NCC}}$ & $\overline{\mathrm{N} N \overline{\mathrm{NCN} N C}}$ & N $\overline{\mathrm{NNCCN}}$ & NNNDNNN \\
\hline \multirow[t]{2}{*}{ [NNNCCC] } & $\mathrm{U}$ & 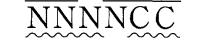 & N & & NNN \\
\hline & & NNNNNC & NNNNNC & & NNC NNN \\
\hline$Q_{13}(+B++)$ & $\mathrm{E}$ & $\overline{\mathrm{NNCN}} \mathrm{N} \overline{\mathrm{C}}$ & $\overline{\text { NCN NN}} \bar{\sim} \overline{N C}$ & N $\overline{N N} N N \bar{N}$ & $\mathrm{~N} \overline{\mathrm{CCNNN}}$ \\
\hline \multirow[t]{2}{*}{ [NCCNNC] } & $\mathrm{U}$ & & NC NNNC & NNNNCN & \\
\hline & & & NNNNNC & $\mathrm{NNNCCN}$ & \\
\hline$Q_{14}(++C+)$ & $E$ & $\overline{\mathrm{NNCNC}}$ & $\overline{\mathrm{N}} N \bar{\sim} \overline{\mathrm{N}} N \bar{\sim} \mathrm{N} N$ & $\overline{\mathrm{CNN}} \mathrm{N} \overline{\mathrm{CN}}$ & $\overline{\mathrm{C}} \mathrm{N} \overline{\mathrm{CNNN}}$ \\
\hline \multirow{2}{*}[\mathrm{CNCNCN}]{} & $\mathrm{U}$ & $\overline{\text { NNCNCN }}$ & & & $\overline{\mathrm{C}} \mathrm{N} \overline{\mathrm{CNNN}}$ \\
\hline & & NNC NNN & & & NNCNNN \\
\hline$Q_{15}(+++D)$ & $\mathrm{E}$ & $\overline{\mathrm{NN}} \mathrm{N} \overline{\mathrm{N}} \mathrm{NN}$ & $\overline{\mathrm{NCNCNN}}$ & $\overline{\mathrm{CNNC}} \mathrm{NN}$ & $\overline{\mathrm{CC}} \mathrm{N} \overline{\mathrm{NNN}}$ \\
\hline \multirow{2}{*}[\mathrm{CCNCNN}]{} & $\mathrm{U}$ & & NCNCNN & CNNCNN & $\overline{\mathrm{CC}} \mathrm{NNNN}$ \\
\hline & & & NCNNNN & NNNCNN & NCNNNN \\
\hline
\end{tabular}

On U, only cases which are different from $\mathrm{E}$ are described.

N means that these N's cannot be explained by the donor or receptor.

(See Table 1 for explanation, also.)

Table 5. Influence of fitness values given in each virulence gene on type of nonrandom associations $\left(\mathrm{S}_{2} \rightarrow \mathrm{S}_{3}\right)$

\begin{tabular}{|c|c|c|c|c|c|}
\hline \multirow{2}{*}{$\begin{array}{c}\text { Donor } \\
\text { (Genotype) }\end{array}$} & & \multicolumn{4}{|c|}{ Receptor } \\
\hline & & $\begin{array}{l}Q_{12}(\mathrm{~A}+++) \\
{[\mathrm{CCCNNN}]}\end{array}$ & $\begin{array}{l}Q_{13}(+\mathrm{B}++) \\
{[\mathrm{CNNCCN}]}\end{array}$ & $\begin{array}{l}Q_{14}(++\mathrm{C}+) \\
{[\mathrm{NCNCNC}]}\end{array}$ & $\begin{array}{l}Q_{15}(+++\mathrm{D}) \\
{[\mathrm{NNCNCC}]}\end{array}$ \\
\hline $\begin{array}{l}Q_{6}(\mathrm{AB}++) \\
{[\mathrm{CNNNNC}]}\end{array}$ & $\mathrm{E}$ & 1. $\underline{\mathrm{CCCNNN}}$ & 2. $\underline{\mathrm{CNNCCN}}$ & 3. C $\underline{C N C N C}$ & 4. C NCNCC \\
\hline $\begin{array}{l}Q_{7}(\mathrm{~A}+\mathrm{C}+) \\
{[\mathrm{NCNNCN}]}\end{array}$ & $\begin{array}{l}\mathrm{E} \\
\mathrm{U}\end{array}$ & 5. $\mathrm{CCCNNN}$ & 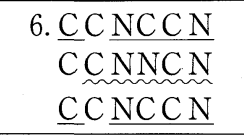 & 7. $\mathrm{NCNCNC}$ & 8. $\underline{\mathrm{NCCNCC}}$ \\
\hline $\begin{array}{l}Q_{8}(+\mathrm{BC}+) \\
{[\mathrm{NNCCNN}]}\end{array}$ & $\mathrm{E}$ & 9. $\mathrm{CCCCNN}$ & 10. $\mathrm{CNNCCN}$ & 11. $\mathrm{NCNCNC}$ & 12. $\mathrm{NNCCCC}$ \\
\hline $\begin{array}{l}Q_{9}(\mathrm{~A}++\mathrm{D}) \\
{[\mathrm{NNCCNN}]}\end{array}$ & $\mathrm{E}$ & 13. $\mathrm{CCCNNN}$ & 14. $\underline{\mathrm{CNCCCN}}$ & 15. NCCCNC & 16. $\mathrm{NNCNCC}$ \\
\hline $\begin{array}{l}Q_{10}(+\mathrm{B}+\mathrm{D}) \\
{[\mathrm{NCNNCN}]}\end{array}$ & $\begin{array}{l}\mathrm{E} \\
\mathrm{U}\end{array}$ & $\begin{array}{l}\text { 17. } \frac{\mathrm{CCCNCN}}{\mathrm{CCNNCN}} \\
\underline{\mathrm{CCCNCN}}\end{array}$ & 18. $\mathrm{CNNCCN}$ & 19. $\frac{\text { NC NCCC }}{\text { NCNCCN }}$ & 20. NNC NCC \\
\hline $\begin{array}{l}Q_{11}(++\mathrm{CD}) \\
{[\mathrm{CNNNNC}]}\end{array}$ & $\begin{array}{l}\mathrm{E} \\
\mathrm{U}\end{array}$ & 21. $\frac{\mathrm{CCCNNC}}{\mathrm{CCNNNC}}$ & 22. $\frac{\text { CNNCCC }}{\text { CNNNNC }}$ & 23. NC NC NC & 24. $\mathrm{NNC} \mathrm{NCC}$ \\
\hline
\end{tabular}

On $\mathrm{U}$, only cases which are different from $\mathrm{E}$ are described.

(See Table 1 for explanation, also.)

line, two of four host-genotype combinations show $6 \mathrm{R}$ patterns and the others show $5 \mathrm{R}$ patterns. $6 \mathrm{R}$ and $5 \mathrm{R}$ appear where the number of complementary loci is one and three, respectively. Pairs different from the $6 \mathrm{R}$ pattern in the latter are always pairs of avirulence loci corresponding to resistance genes in the donor. For example, when a donor is $\mathrm{A}+\mathrm{C}+$, pairs of a and $\mathrm{c}$ show a donor type of nonrandom association.

In reverse transfer $\left(S_{3} \rightarrow S_{2}\right)$ (Table 6), half of hostgenotype combinations in each line show $6 \mathrm{D}$ patterns and the others are $5 \mathrm{D}$ patterns under the standard conditions. In these cases, $6 \mathrm{D}$ patterns change to $5 \mathrm{D}$ patterns with generations. These $6 \mathrm{D}$ patterns are limited to cases where one locus is complementary ; $5 \mathrm{D}$ patterns appear where three loci are complementary.

Change in nonrandom association patterns when a large value is given to one host genotype

A concrete solution has not been obtained in simulation studies up to now on the first question of why there were always two kinds of nonrandom associations in all 
Table 6. Influence of fitness values given in each virulence gene on type of nonrandom associations $\left(\mathrm{S}_{3} \rightarrow \mathrm{S}_{2}\right)$

\begin{tabular}{|c|c|c|c|c|c|c|c|}
\hline \multirow[b]{2}{*}{$\begin{array}{c}\text { Donor } \\
\text { (Genotype) }\end{array}$} & & \multicolumn{6}{|c|}{ Receptor } \\
\hline & & $\begin{array}{c}Q_{6}(\mathrm{AB}++) \\
{[\mathrm{NCCCCN}]}\end{array}$ & $\begin{array}{c}Q_{7}(\mathrm{~A}+\mathrm{C}+) \\
{[\mathrm{CNC} \mathrm{NC}]}\end{array}$ & $\begin{array}{c}Q_{8}(+\mathrm{BC}+) \\
{[\mathrm{CCNNCC}]}\end{array}$ & $\begin{array}{c}Q_{9}(\mathrm{~A}++\mathrm{D}) \\
{[\mathrm{CCNNCC}]}\end{array}$ & $\begin{array}{l}Q_{10}(+B+D) \\
{[C N C C N C]}\end{array}$ & $\begin{array}{l}Q_{11}(++C D) \\
{[N C C C C N]}\end{array}$ \\
\hline $\begin{array}{l}Q_{12} \\
(\mathrm{~A}+++)\end{array}$ & $\mathrm{E}$ & NNNCCC & $\underbrace{\text { NNNCNC }}_{\text {NNNCNCCC }}$ & NNNNCC & $\begin{array}{l}\text { NNNCCC } \\
\text { NNNNCC }\end{array}$ & NNNCNC & NNNCCN \\
\hline$[\mathrm{NNNCCC}]$ & U & & & & & $\mathrm{NNCCNC}$ & $\begin{array}{l}\mathrm{NNCCCN} \\
\text { NCCCCN } \\
\end{array}$ \\
\hline $\begin{array}{l}Q_{13} \\
(+\mathrm{B}++)\end{array}$ & $\mathrm{E}$ & $\begin{array}{l}\text { NCCNNC } \\
\text { NCCNNN }\end{array}$ & NNC NNC & $\begin{array}{l}\text { NCCNNC } \\
\text { NCNNNC }\end{array}$ & NCNNNC & $\begin{array}{l}\text { NCCNNC } \\
\text { NNC NNC }\end{array}$ & NCCNNN \\
\hline$[\mathrm{NCCNNC}]$ & $\mathrm{U}$ & & $\begin{array}{l}\mathrm{NNCCNC} \\
\mathrm{CNCCNC} \\
\end{array}$ & & $\begin{array}{l}\mathrm{NCNNCC} \\
\text { CCNNCC }\end{array}$ & & $\underline{N} \underline{C C C N}$ \\
\hline $\begin{array}{l}Q_{14} \\
(++C+)\end{array}$ & $\mathrm{E}$ & NNCNCN & CNCNCNNN & CNCNCN & CNNNCN & CNCNNN & $\begin{array}{l}\text { CNCNCN } \\
\text { NNCNCN }\end{array}$ \\
\hline$[\mathrm{CNCNCN}]$ & $\mathrm{U}$ & & & & $\begin{array}{l}\text { CNNNCN } \\
\text { CNNNCC } \\
\underline{C}\end{array}$ & & \\
\hline $\begin{array}{l}Q_{15} \\
(+++D)\end{array}$ & $\mathrm{E}$ & NCNCNN & CNNCNN & CCNNNN & $\begin{array}{l}\text { CCNCNN } \\
\text { CCNNNN }\end{array}$ & $\begin{array}{l}\text { CCNCNN } \\
\text { CNNCNN }\end{array}$ & $\begin{array}{l}\text { CCNCNN } \\
\text { NCNCNN }\end{array}$ \\
\hline$[\mathrm{CCNCNN}]$ & U & & & & $\underset{\text { CDNNCNN }}{\text { CCNCNN }}$ & CCNCNN & CCNCNN \\
\hline
\end{tabular}

On $\mathrm{U}$, only cases which are different from $\mathrm{E}$ are described.

(See Table 1 for explanation, also).

Table 7. Nonrandom associations when one gene of four was added in an area where 16 genotypes are present under the equilibrium conditions

\begin{tabular}{|c|c|c|c|c|c|c|c|c|c|c|}
\hline \multirow{2}{*}{$\begin{array}{c}\begin{array}{c}\text { Genotype } \\
\text { added }\end{array} \\
\mathrm{A}+++\end{array}$} & \multicolumn{4}{|c|}{$\begin{array}{l}\text { Fitness values } \\
\text { given to }+^{\mathrm{d}},+^{\mathrm{c}} \text {, } \\
+^{\mathrm{b}} \text { and }+^{\mathrm{a}}\end{array}$} & \multicolumn{6}{|c|}{$\begin{array}{l}\text { Nonrandom association pattern and } \\
\text { avirulence locus combination it occurs }\end{array}$} \\
\hline & 0.8 & 0.8 & 0.8 & 0.8 & \multirow{5}{*}{$\begin{array}{c}\mathrm{C} \\
\underline{\mathrm{a}}-\mathrm{b}\end{array}$} & \multirow[b]{3}{*}{$\mathrm{C}$} & \multirow[b]{3}{*}{$\mathrm{C}$} & \multirow[b]{3}{*}{$\mathrm{N}$} & \multirow{5}{*}{$\begin{array}{c}\mathrm{N} \\
\mathrm{b}-\mathrm{d}\end{array}$} & \multirow{5}{*}{$\begin{array}{l}\mathrm{N} \\
\mathrm{c}-\mathrm{d}\end{array}$} \\
\hline & 0.9 & 0.8 & 0.6 & 0.7 & & & & & & \\
\hline & 0.8 & 0.6 & 0.7 & 0.9 & & & & & & \\
\hline & 0.6 & 0.8 & 0.9 & 0.7 & & $\underline{a}^{-c}$ & $\underline{\mathrm{a}}-\mathrm{d}$ & $b-c$ & & \\
\hline & 0.6 & 0.9 & 0.7 & 0.8 & & & & & & \\
\hline \multirow{2}{*}{$+\mathrm{B}++$} & 0.8 & 0.8 & 0.8 & 0.8 & $\mathrm{C}$ & $\mathrm{N}$ & $\mathrm{N}$ & $\mathrm{C}$ & $\mathrm{C}$ & $\mathrm{N}$ \\
\hline & & & & & $a-\underline{b}$ & $a-c$ & $a-d$ & $\underline{b}-c$ & $\underline{b}-d$ & $c^{-} d$ \\
\hline \multirow{2}{*}{$++\mathrm{C}+$} & 0.8 & 0.8 & 0.8 & 0.8 & $\mathrm{~N}$ & $\mathrm{C}$ & $\mathrm{N}$ & $\mathrm{C}$ & $\mathrm{N}$ & $\mathrm{C}$ \\
\hline & & & & & $a-b$ & $\mathrm{a}-\underline{\mathrm{c}}$ & $a-d$ & $\mathrm{~b}-\underline{\mathrm{c}}$ & $\mathrm{b}-\mathrm{d}$ & $\underline{c}-d$ \\
\hline \multirow[t]{2}{*}{$\mathrm{AB}++$} & 0.8 & 0.8 & 0.8 & 0.8 & $\mathrm{~N}$ & $\mathrm{C}$ & $\mathrm{C}$ & $\mathrm{C}$ & $\mathrm{C}$ & $\mathrm{N}$ \\
\hline & & & & & $\underset{\approx-b}{a-b}$ & $\underline{\mathrm{a}}-\mathrm{c}$ & $\underline{\mathrm{a}}^{-\mathrm{d}}$ & $\underline{b}-c$ & $\underline{b}-d$ & $c^{-}-d$ \\
\hline \multirow{2}{*}{$+\mathrm{B}+\mathrm{D}$} & 0.8 & 0.8 & 0.8 & 0.8 & $\mathrm{C}$ & $\mathrm{N}$ & $\mathrm{C}$ & $\mathrm{C}$ & $\mathrm{N}$ & $\mathrm{C}$ \\
\hline & & & & & $a-\underline{b}$ & $a^{-} c$ & $\mathrm{a}-\underline{\mathrm{d}}$ & $\underline{\mathrm{b}}^{-\mathrm{c}}$ & $\underset{\approx}{\mathrm{b}-\mathrm{d}}$ & $c^{-} \underline{d}$ \\
\hline \multirow[t]{2}{*}{$\mathrm{ABC}+$} & 0.8 & 0.8 & 0.8 & 0.8 & $\mathrm{~N}$ & $\mathrm{~N}$ & $\mathrm{C}$ & $\mathrm{N}$ & $\mathrm{C}$ & $\mathrm{C}$ \\
\hline & & & & & $\underset{\sim}{a-b}$ & $\stackrel{a-c}{\approx}$ & $\underline{a}-d$ & b-c & $\underline{\mathrm{b}}-\mathrm{d}$ & $\underline{c}-d$ \\
\hline
\end{tabular}

indicates that a host with one resistance gene induces cross-type nonrandom association in combinations singly having the avirulence gene corresponding to the resistance gene.

$\approx \approx$ indicates that a host with two resistance genes induces noncross-type nonrandom associations in combinations together having the avirulence genes corresponding to the resistance genes.

fields where two or more nonrandom associations were found in a field where blast fungus was collected from a resistant variety. Therefore, an attempt was made to get change in nonrandom association types and patterns where one area is largely covered by one genotype.
Simulations began from equilibrium conditions. The results are shown in Table 7 . In these cases, 0.2 were added to some genotypes, $\mathrm{A}+++,+\mathrm{B}++,++\mathrm{C}+$, $\mathrm{AB}++,+\mathrm{B}+\mathrm{D}$ and $\mathrm{ABC}+$, separately. Simulations began after making the total 1.0. In the case of $\mathrm{A}+++$, 
five sets of fitness values were given to the four virulence genes. Results obtained for these five sets are the same. When $\mathrm{A}+++$ genotype was added, pairs including the avirulence locus, a, corresponding to the resistance gene added always showed $\mathrm{C}$ type nonrandom associations. Pairs without the avirulence gene, a, always show N type nonrandom associations in a range of the use of genotypes including that of only one gene. When genotypes including two or more resistance genes were added, pairs of two avirulence loci (marked with $\approx \approx$ in the table) corresponding to resistance genes added showed $\mathrm{N}$ type nonrandom associations. Thus, a ratio of $\mathrm{C}: \mathrm{N}$ was $3: 3$ in the cases where one or three resistance genes were added; it was $4: 2$ in the cases of two resistance genes. This indicates that the probability that both types are included in two cases selected is very high. Accordingly, the fact that two nonrandom association types $(\mathrm{C}$ and $\mathrm{N})$ were found in each of three fields where two or more statistically significant nonrandom associations were found is a natural phenomenon, not due to chance.

\section{GENERAL DISCUSSION}

The results of the present paper are adjusted in Table 8 with the results in the previous paper ${ }^{6}$. The following principles were proposed in the previous paper ${ }^{6)}$. That is, nonrandom association types are determined by donor or receptor. When the donor determines them, combinations of a resistance gene and a susceptibility gene show cross-type $(\mathrm{N})$ nonrandom association.

When the receptor determines them, reverse types are induced. These principles can be applied to combinations among different $\mathrm{S}$ groups (Table 8). In this table, the predominant pattern of nonrandom associations in each group combination (line in the table) are shown. Although almost all cases could be explained by the types of donor or receptor, there are some cases which cannot be explained thus. In almost all lines, pairs of avirulence loci which cannot be explained by the donor or receptor, show only one type (C or $\mathrm{N})$ in each line. Only in two lines $\left(\mathrm{S}_{2} \rightarrow \mathrm{S}_{4}\right.$ and $\left.\mathrm{S}_{4} \rightarrow \mathrm{S}_{2}\right)$, two types, $\mathrm{N}$ and $\mathrm{C}$, are found to occupy the pairs which cannot be explained by the donor or receptor.

The following factors are considered to play a large role in determining nonrandom association types and patterns in the described order : 1 ) direction of replacement of two host genotypes (transfer of value corresponding to frequency of the genotypes), 2) combinations between groups of genotypes with the same number of susceptibility genes, 3) transferred values (area of replaced host genotypes), 4) generations of vegetative reproduction, and 5) the number of resistance loci with complementary resistance and susceptibility genes.

This series of studies was started from the finding that there were two types, $\mathrm{C}$ and $\mathrm{N}$, in nonrandom associations between two avirulence loci per field, in each of the fields where two or more statistically significant nonran-
Table 8 . Summary of results of simulations

\begin{tabular}{|c|c|c|c|c|}
\hline \multirow{2}{*}{$\begin{array}{l}\text { Number of } \\
\text { susceptibility } \\
\text { genes }^{\text {a) }}\end{array}$} & \multicolumn{4}{|c|}{ Number of complementary genes } \\
\hline & 1 & 2 & 3 & 4 \\
\hline \multicolumn{5}{|l|}{ Increase } \\
\hline $\mathrm{S}_{0} \rightarrow \mathrm{S}_{1}$ & $6 \mathrm{R}$ & & & \\
\hline $\mathrm{S}_{0} \rightarrow \mathrm{S}_{2}$ & & $6 \mathrm{R}$ & & \\
\hline $\mathrm{S}_{0} \rightarrow \mathrm{S}_{3}$ & & & $6 \mathrm{D}$ & \\
\hline $\mathrm{S}_{0} \rightarrow \mathrm{S}_{4}$ & & & & $6 \mathrm{D}$ \\
\hline $\mathrm{S}_{1} \rightarrow \mathrm{S}_{2}$ & $6 \mathrm{R}$ & & $5 \mathrm{R}^{\mathrm{b})}$ & \\
\hline $\mathrm{S}_{1} \rightarrow \mathrm{S}_{3}$ & & 5DR C & & 3DR C \\
\hline $\mathrm{S}_{1} \rightarrow \mathrm{S}_{4}$ & & & $6 \mathrm{D} \rightarrow 6 \mathrm{R}^{\mathrm{c})}$ & \\
\hline $\mathrm{S}_{2} \rightarrow \mathrm{S}_{3}$ & $6 \mathrm{R}$ & & $5 \mathrm{R} \mathrm{C}$ & \\
\hline $\mathrm{S}_{2} \rightarrow \mathrm{S}_{4}$ & & $\begin{array}{l}5 \mathrm{DR} \rightarrow \\
6 \mathrm{R} \mathrm{NC} \text { ) }\end{array}$ & & \\
\hline $\mathrm{S}_{3} \rightarrow \mathrm{S}_{4}$ & $6 \mathrm{D}$ & & & \\
\hline \multicolumn{5}{|l|}{ No change } \\
\hline $\mathrm{S}_{1} \rightarrow \mathrm{S}_{1}$ & & $6 \mathrm{R}$ & & \\
\hline $\mathrm{S}_{2} \rightarrow \mathrm{S}_{2}$ & & $6 \mathrm{R}$ & & $5 \mathrm{R} \mathrm{C}$ \\
\hline $\mathrm{S}_{3} \rightarrow \mathrm{S}_{3}$ & & $\begin{array}{l}5 \mathrm{DR} \rightarrow \\
6 \mathrm{R} \mathrm{N}\end{array}$ & & \\
\hline \multicolumn{5}{|l|}{ Decrease } \\
\hline $\mathrm{S}_{1} \rightarrow \mathrm{S}_{0}$ & $6 \mathrm{D}$ & & & \\
\hline $\mathrm{S}_{2} \rightarrow \mathrm{S}_{0}$ & & $5 \mathrm{D} \mathrm{N}$ & & \\
\hline $\mathrm{S}_{3} \rightarrow \mathrm{S}_{0}$ & & & $6 \mathrm{R}$ & \\
\hline $\mathrm{S}_{4} \rightarrow \mathrm{S}_{0}$ & & & & $6 \mathrm{R}$ \\
\hline $\mathrm{S}_{2} \rightarrow \mathrm{S}_{1}$ & $6 \mathrm{D}$ & & $5 \mathrm{D} \rightarrow 6 \mathrm{R} \mathrm{N}$ & \\
\hline $\mathrm{S}_{3} \rightarrow \mathrm{S}_{1}$ & & $5 \mathrm{DR} N$ & & $3 \mathrm{DR} N$ \\
\hline $\mathrm{S}_{4} \rightarrow \mathrm{S}_{1}$ & & & $6 \mathrm{R}$ & \\
\hline $\mathrm{S}_{3} \rightarrow \mathrm{S}_{2}$ & $6 \mathrm{D} \rightarrow 5 \mathrm{D} \mathrm{N}$ & & $5 \mathrm{DR} N$ & \\
\hline $\begin{array}{l}\mathrm{S}_{4} \rightarrow \mathrm{S}_{2} \\
\mathrm{~S}_{4} \rightarrow \mathrm{S}_{3}\end{array}$ & $6 \mathrm{D} \rightarrow 6 \mathrm{R}$ & 5DR NC & & \\
\hline
\end{tabular}

a) "Increase", "No change" and "Decrease" indicate increase or decrease in the number of susceptibility genes at transferring of values (frequencies)

b) In $5 \mathrm{D}$ or $5 \mathrm{R}$, pairs of which observed patterns do not agree with patterns expected from the donor or receptor always show only $\mathrm{N}$ or $\mathrm{C}$ type in the combination of $S$ groups.

c) Indicating that patterns change from the former pattern to the latter pattern with generations.

d) There are both $\mathrm{N}$ and $\mathrm{C}$ types in pairs of which are not in agreement with the donor or receptor patterns in the same group combination.

For example, CNNNNN $\left(Q_{6} \rightarrow Q_{16}\right)$ and $\operatorname{NCCCCC~}\left(Q_{16}\right.$ $\rightarrow Q_{6}$ ) in Table 3.

dom associations were found. We improved upon the simulation program made by Kiyosawa ${ }^{1}$ and Kiyosawa et $a l .{ }^{2,3)}$ to simulate the reproduction of pathogen genotypes in the four gene system of disease resistance. Simulations always began from equilibrium conditions. This makes interpretation of the results easy.

In individual pairs of avirulence loci, in some cases receptors determine the type of nonrandom associations, and in other cases, donors determine them. When receptors determine the nonrandom association types $(6 \mathrm{R})$, pairs of resistance genes or susceptibility genes induce $\mathrm{N}$ type nonrandom associations, and pairs of a resist- 
ance gene and a susceptibility gene induce the $\mathrm{C}$ types. When donors determine the types (6D), the types of nonrandom associations induce the reverse of those which receptors determine. Frequently, there are intermediate patterns. In some of these cases, five out of six pairs are explained by the donor type and the other five are explained by the receptor type (5DR). Nonrandom association types and patterns change by the fitness values given to the virulence genes concerned, and in some cases also by vegetative generations. When the types and patterns change with generation, the change is from donor type to receptor type.

In the first paper of this series ${ }^{4)}$, we noticed the two types in two or three nonrandom associations found in a field. This high probability cannot necessarily be explained by the results of the simulations mentioned above. Therefore, a larger value was given to a certain host genotype in simulations, that is, a larger area was practically replaced by a new variety having a different gene. This is a more likely situation in former's fields. The results of simulations under such situations showed that half of six pairs among the avirulence loci are $\mathrm{N}$ type and the other half are $\mathrm{C}$ type when the new variety has one or three genes for resistance. This can explain the phenomena that two types always appear in two or three nonrandom associations found in a field in three cases $^{4}$.

Thus, this series of simulations clarified that the presence of two nonrandom associations in a field where varieties with some resistance gene(s) are cultivated ${ }^{4)}$ is a natural (or general) phenomenon and not a result of chance.

We recently obtained the result, by simulation experiments, that when different values were given for fitness of virulence genes, different nonrandom association types and patterns could be noticed ${ }^{5,6}$. These results suggest the possibility that an approximate estimate of fitness (cost) of virulence genes can be obtained by virulence analyses of field data on the distribution of pathogen genotypes. Including this one, virulence analysis seems useful to analyze the structure of hostpathogen populations and to estimate fitness values.

\section{Literature cited}

1. Kiyosawa, S. (1986). Estimation of stabilizing selection of virulence genes in the rice blast fungus population in prefectures of Japan. Bull. Natl. Inst. Agrobiol. Resour. $2: 61-91$

2. Kiyosawa, S., Fujimaki, Y. and Iwano, M. (1993). Collections of blast fungus from resistant rice varieties and their frequencies of races and virulence genes. Ann.
Phytopathol. Soc. Jpn. 59 : 387-395.

3. Kiyosawa, S., Fujimaki, Y. and Iwano, M. (1993). Estimation of fitness values for virulence genes in collections of rice blast fungus from Niigata. Ann. Phytopathol. Soc. Jpn. 59 : 396-406.

4. Kiyosawa, S., Okinaka, Y., Purba, D. and Furutani, F. (1994). Collections of blast fungus from resistant rice varieties and their virulence analysis. Breed. Sci. 44 : 419-425.

5. Kiyosawa, S., Purba, D., Ali, M.S., Okinaka, Y., Shimizu, T. and Saito, A. (1995). Influence of frequencies of host genotypes and fitness of virulence genes on nonrandom association type and pattern between avirulence loci. Breed. Sci. $45:$ 287-293.

6. Kiyosawa, S., Purba, D., Ali, M.S., Okinaka, Y., Shimizu, T. and Saito, A. (1996). Nonrandom association of avirulence loci in four disease resistance gene system. I. Principles of determination of nonrandom association type and pattern and its confirmation within groups having the same number of susceptibility genes. Ann. Phytopathol. Soc. Jpn. $62: 95-100$.

\section{和 文 摘 要}

清沢茂久 - Donna PURBA - Md. Shamsher ALI ・沖中 泰 ・ 清水 勉・斉藤明彦: 4 病害抵抗性遺伝子系におりる非病原性 遺伝子座の非機会的結合 II. 異なった数の罹病性遺伝子を持 つた宿主群間の品種の交替

抵抗性遺伝子座間の同一罹病性遺伝子数を持つ群内の組合せ に関して, 非病原性遺伝子座間の非機会的結合について前報で 提案した原理が，その他の組合せにも適用できるかをシミュレ ーションの方法で検討した。その結果, 前報の結果も含めて次の ことが明らかにされた。宿主遺伝子型の切り替えにより起こる 病原菌の非病原性遺伝子に関する非機会的結合には, 供与者 （古い遺伝子型）により決められるときと, 受容者（新しい遺伝 子型)により決められるときとがある。前者では, 抵抗性遺伝子 と罹病性遺伝子との組合せで非交差 $(\mathrm{N})$ 型の非機会的結合が生 じ，他は交差 (C) 型の非機会的結合を示す。種々の S 群 (同一 数の罹病性遺伝子を持つ遺伝子型の群) の組合せの非機会的結 合の型やパターンは概ね上記の原理に基づいて決まるが, 種々 の要因によって変化を見せる。非機会的結合パターンを変える ものは, 上述の, 1) 宿主遺伝子型の切り替えの方向が最も大き な影響を与え, それ以外に, 2) 罹病性遺伝子の数で分類した群 の組合せ, 3）個々の病原性遺伝子の適応値, 4) 抵抗性遺伝子と 罹病性遺伝子が相補的に存在する遺伝子座の数, などがパター ンの変化をもたらす。種々の遺伝子型, 例えば $\mathrm{A}+++$ +を大量に 作ると宿主内の $\mathrm{A}$ 抵抗性遺伝子に対応する $\mathrm{a}$ 非病原性遺伝子 の関連した対 $a-b, a-c, a-d$ は非交差型の非機会的結合を，他の 対 $\mathrm{b}-\mathrm{c}, \mathrm{b}-\mathrm{d}, \mathrm{c}-\mathrm{d}$ は交差型となる。この場合 $\mathrm{N}: \mathrm{C}$ は $1: 1$ とな り,この一連の研究の目的であった抵抗性品種から採取した菌 の $\mathrm{N}: \mathrm{C}$ 比が $1: 1$ かそれに近かったことを十分に説明し得る ものと考えられる。 